\title{
Foreign body in the airway of a premature infant: a fragment of suction catheter revisited
}

\author{
Aybuke Yazici*, Betül S Bilgin and Sevim Unal \\ Division of Neonatology, Health Science University, Ankara Children's Hematology and Oncology Research Hospital, Turkey
}

\begin{abstract}
Paediatric foreign body in the airway is usually seen in children aged 1-3 years and cause sudden respiratory distress. If the diagnosis is delayed or untreated, it may result in serious morbidity or even mortality. In new-borns, foreign body in the airways is rarely seen and it is usually formula or breast milk. We presented a premature hypotonic infant in need of mechanical ventilation transported to our unit on 49th day for tracheotomy. We identified left pulmonary atelectasis and a plastic tube fragment was removed from the left main bronchus by rigid bronchoscopy.
\end{abstract}

\section{Introduction}

Paediatric foreign body in the airway (FBA) is usually seen in children aged 1-3 years and cause sudden respiratory distress. If the diagnosis is delayed or untreated, it may result in serious morbidity or even mortality. In new-borns, FBA is rare and usually develop secondary to formula or breast milk aspiration $[1,2]$. Even performed by a skilled health care personal, tracheal suctioning might cause adverse effects and severe complications such as tracheal tube dislocation, bradycardia, atelectasis, pneumothorax, and very rarely tube obstruction by a foreign body [3]. The tracheal tube obstruction by a fragment of suction catheter was first described by Meneghetti in 1996 [4]. We presented a premature hypotonic infant referred to our hospital for tracheotomy. There was left pulmonary atelectasis and a 3 $\mathrm{cm}$ feeding tube fragment was removed from the left main bronchus via bronchoscopy that resulted in full recovery.

\section{Case report}

A premature infant born at 30 weeks old and $1450 \mathrm{~g}$ was referred to our hospital for tracheotomy on 49th days old. The baby was on conventional mechanical ventilation (CMV) from birth, as she was diagnosed with hypotonic infant, and could not be weaned. There were peripheral hypotonia, club foot, increased deep tendon reflexes and decreased breath sounds in the left hemithorax. We defined infiltration in the right lower lobe, left pulmonary and right linear atelectasis in her chest X-ray (Figure 1).

The patient applied CMV, piperacillin-tazobactam and amikacin with the diagnosis of nosocomial pneumonia. On the 6th day of
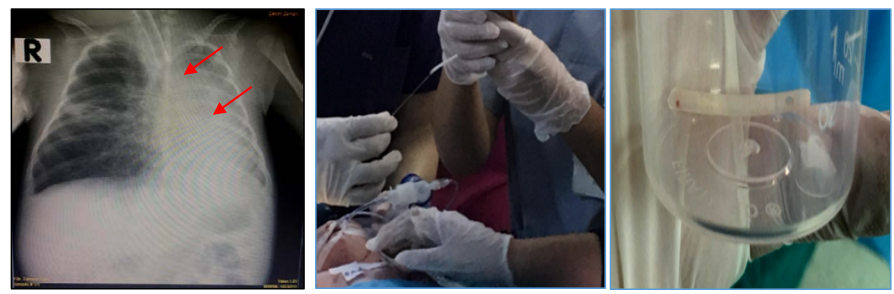

Figure 1. The chest $\mathrm{x}$-ray on admission and about $3 \mathrm{~cm}$ suction catheter fragment removed from the left main bronchus hospitalization, tracheotomy was performed, and atelectasis did not improve. The respiratory tract was evaluated by flexible laryngoscopy and there was an abnormal appearance in the left main bronchus. Rigid bronchoscopy was performed and about $3 \mathrm{~cm}$ suction catheter fragment with two side-holes was removed from the left main bronchus (Figure 1 ). When the chest $\mathrm{x}$-ray on admission was revaluated, we noticed an about $2-3 \mathrm{~cm}$ hardly visible radiopaque line in the left main bronchus. Following the removal of suction catheter fragment and revision of the antibiotic therapy, pneumonia and atelectasis were improved. She was re-transported to local hospital close to her family one month later.

\section{Discussion and conclusion}

To date, lamp of laryngoscope, sheath of tracheal tube style, aspiration catheter tip (usually closed tracheal suction system) and very rarely endotracheal tube in the airway was reported in neonates needing artificial respiration $[1,2,4-7]$. Herein, we present a premature hypotonic infant in need of CMV and a fragment of suction catheter was removed by rigid bronchoscopy. We thought that she has been applied intermittent respiratory care, and secretions within the respiratory system has been occasionally aspirated due to the long-term CMV need. We suggested that this foreign body might be a fragment of a closed tracheal suction catheter and rupture of the catheter was undetected after aspiration by high pressure.

Foreign body in the airway may cause chronic lung disease, apart from acute respiratory distress. In the early period, FBA causes shortness of breath, cough, wheezing, unilateral decrease in breath sounds, hoarseness. History of FBA may not be clear, symptoms may occur in a sudden or prolonged period, physical examination and radiological findings may be normal. ${ }^{1,2}$ Besides, FBA are $90 \%$ non-radiopaque and

${ }^{\star}$ Correspondence to: Aybüke Yazici, Division of Neonatology, Health Science University, Ankara Children's Hematology and Oncology Research Hospital, Turkey, Tel: +90 312 5969730; Fax: +90 312 3472330; E-mail: dr.a.yazici@ hotmail.com

Key words: feeding tube, foreign body, airway, newborn

Received: April 22, 2019; Accepted: April 13, 2019; Published: May 17, 2019 
are difficult to detect. This may cause a delay in diagnosis and treatment and high complication ratio. In the late period, complications such as pneumonia, atelectasis, emphysema, lung abscess, broncho oesophageal fistula, empyema and bronchiectasis may develop. In case of chronic respiratory symptoms or development of unilateral pulmonary findings, physicians should keep in mind FBA. The clinical suspicion is essential in the diagnosis of FBA and foreign body must be removed by bronchoscopy [2]. If an experienced team perform bronchoscopy with appropriate equipment in the proper conditions, the complication rate would be low and the success rate would be high [1,2]. History and physical findings were not suggested FBA in this case. Therefore, we want to underline that clinical suspicion is important in diagnosis of FBA and x-rays should be carefully evaluated not to overlooked radiopaque foreign bodies.

In conclusion, FBA is rarely seen in new-borns. On the other hand, FBA should be suspected in a new born with chronic respiratory symptoms, unilateral radiologic findings, especially with atelectasis and $\mathrm{x}$-rays should be carefully evaluated. It was emphasized that health care professionals should be careful during aspiration, respiratory care, intubation and CMV in the infants. Following aspiration, they would not forget to check the closed suction catheter length and integrity in terms of rupture or separation.

\section{References}

1. Shubha MA, Das K (2009) Tracheobronchial foreign bodies in infants. Int J Pediatric Otorhinolaryngol 73: 1385-1389. [Crossref]

2. Hegde SV, Hui PKT, Lee EY (2014) Tracheobronchial foreign bodies in children Imaging assessment. Semin Ultrasound CT MRI 36: 8-20. [Crossref]

3. Sawyera T, Fogliab E, Hatche LD, Moussad A, Adesb A, et al. (2017) Improving neonatal intubation safety: A journey of a thousand miles. J Neonatal Perinatal Med 10: 125-131. [Crossref]

4. Meneghetti S, Trevisanuto D, Cantarutti F, Zanardo V (1996) Tracheal tube obstruction by suction catheter fragment in a premature baby with RDS. Paediatr Anaesth 6: 163164. [Crossref]

5. Nurkin S, Waisman D, Davkin O, Kessel I, Vinograd I, et al. (2004) A rare complication of the closed tracheal suction system. J Pediatr 145: 858. [Crossref]

6. Shah DM, Sinn J (2010) Endotracheal suction catheter as a foreign body in a neonate. J Paediatr Child Health 46: 209-211. [Crossref]

7. Jasinovic T, Thamboo A, Osiovich H, Scheepers L, Robinson A, et al. (2013) Best management of ultra-small tracheobronchial foreign bodies in neonates. Int J Pediatr Otorhinolaryngol 77: 434-438. [Crossref]

Copyright: (C2019 Yazici A. This is an open-access article distributed under the terms of the Creative Commons Attribution License, which permits unrestricted use, distribution, and reproduction in any medium, provided the original author and source are credited. 